\title{
Everyday space weather
}

\author{
Madhulika Guhathakurta* \\ Heliophysics Division, NASA Headquarters/Goddard Space Flight Center, Greenbelt, MD 20771, USA
}

Received 24 February 2021 / Accepted 12 May 2021

Just like our home atmosphere is subject to violent and damaging storms, such as Sandy, our space environment undergoes violent disruptions driven by powerful flares and coronal mass ejections from the Sun. These storms, which define the weather of our space environment, are much more energetic than the storms of terrestrial weather. Space storms are sometimes so explosive that they produce strong shock waves that completely deform the Earth's magnetized atmosphere, the magnetosphere, and ultra-high energy particle radiation that can penetrate shielding to damage satellite electronics and, more important, the health of our astronauts. Furthermore, the effects of space weather are not confined to the space environment, but reach all the way down to the ground to produce the radiation that is dangerous to aircraft and current surges that can disrupt our electric power systems.

September 1, 1859, is sometimes referred to as "the birthday of space weather." It is the day that the Sun unleashed historic solar flares, a rapid sequence of explosions known collectively as "the Carrington Event," after the astronomer Richard Carrington who witnessed with his telescope the extreme whitelight flare. For the next 2 weeks, telegraph lines hummed with geomagnetic electricity, Northern Lights spread as far south as Cuba, and magnetometers around the world went wild as Earth's magnetic field reverberated from the buffeting of powerful solar storms.

Since then, discussion of space weather has been dominated by talk of big events.

For instance: On March 13, 1989 - "the day the Sun brought darkness" - a powerful geomagnetic storm knocked out power across the entire province of Quebec in Canada for more than nine hours (Bolduc, 2002). The storm damaged transformers in Quebec, New Jersey, and Great Britain, and caused more than 200 power anomalies across the USA from the eastern seaboard to the Pacific Northwest. A similar series of "Halloween storms" in October 2003 triggered a regional blackout in southern Sweden and may have damaged transformers in South Africa (Pulkkinen et al., 2005; Gaunt \& Coetzee, 2007).
These are the kind of events that happen every few decades or even centuries. They tend to dominate our planning and research priorities.

Big events, however, are not the only events. Contrary to popular belief, there is such a thing as "everyday space weather," and it is more pervasive than you might suppose (Schrijver, 2015). Consider the following:

\section{The New Heliosphere}

Only about a decade ago, the Sun emerged from the deepest Solar Minimum in a hundred years. During the extreme quiet of 2007-2009, the solar wind became weaker (reduced density, ram pressure and magnetic field strength) than it had been previously in the space age (McComas et al., 2008; Schwadron \& McComas, 2008; Connick et al., 2011; Schwadron et al., 2014a); Coronal Mass Ejections (CMEs) lost their punch (Gopalswamy et al., 2014, 2015); and cosmic rays hit a record high for the Space Age (Mewaldt et al., 2010). We found ourselves living in a "New Heliosphere."

Normalcy was not restored by the 11-year swing of the solar cycle. Instead of a vigorous Solar Maximum, the 2010s brought us a "mini Solar Max," as peculiar in some ways as the extreme Solar Minimum that preceded it (McComas et al., 2013). There have been fewer intense flares, fewer strong geomagnetic storms, and fewer high-energy Solar Energetic Particles (SEPs) than any other Solar Max in modern times. Given these events, there is no reason to think that the decade ahead will be "normal." Indeed, there is some evidence that the present solar Minimum is largely similar to the one in 2007-2009. As the Sun's magnetic field weakens and the solar wind flags, researchers find a significant surge of cosmic rays penetrating the solar system, eclipsing old records (Schwadron et al., 2014a, Schwadron, 2018). In Figure 1 Solar Cycle 24 activity and its impacts on the Earth's space environment is illustrated.

You have experienced "everyday space weather" if. . you have flown onboard a commercial airline.

*Corresponding author: Madhulika.guhathakurta@nasa.gov 


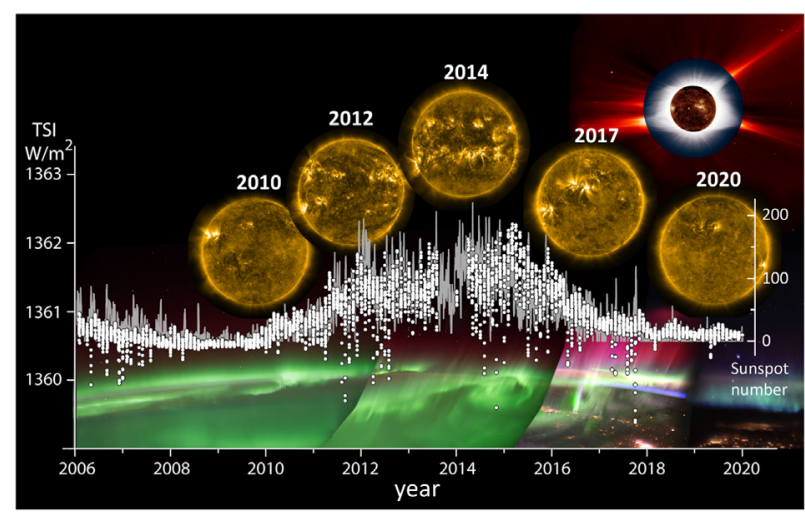

Fig. 1. Illustration of solar activity impacts on the Earth's space environment during Solar Cycle 24. The solar activity increased the Total Solar Irradiance (TSI; white dots) during the solar maximum. Whole-disk observations of the solar corona in the extreme ultraviolet light (Fe IX, $171 \AA$ ) from the Atmospheric Imaging Assembly (AIA) instrument onboard NASA's Solar Dynamics Observatory (SDO) show numerous brightenings caused by magnetic active regions emerging from the solar interior. Aurora images at the bottom are taken from the International Space Station at different solar cycle stages. The upper right corner shows a composite image of the Sun during the total solar eclipse of 2017, at the maximum of solar activity. It includes observations from the NASA instruments LASCO (SOHO mission, in red color), AIA (SDO mission, in brown color), and ground observations of the inner corona by Jay M. Pasachoff.

Flying high above Earth with little atmosphere to protect them, crew and passengers can absorb significant doses of cosmic rays and solar radiation. In any given year, the pilot of your aircraft probably absorbs as much radiation as a worker in a nuclear power plant (Mertens et al., 2016; Tobiska et al., 2016). Passengers flying on commercial aircraft at around 37,000 feet $(\sim 11 \mathrm{~km})$ absorb about 30 times more ionizing radiation than people at sea level. This is the dose rate for midlatitude flights during periods of low solar activity. If you fly over the poles or during a solar storm the radiation dose will be even higher. Typical 100,000 mile frequent fliers absorb as much ionizing radiation as over 10 chest X-rays, and the International Commission on Radiological Protection (ICRP) classifies pilots as "occupational radiation workers."

The source of this radiation is cosmic rays - high energy particles accelerated toward Earth by distant explosions such as supernovas and colliding neutron stars. Cosmic rays penetrate deep inside Earth's atmosphere where airplanes travel every day.

Cosmic Rays are modulated by solar activity in particular by the strength of the solar magnetic field. Just like during the Solar Maximum phase of the solar cycle when the field strength is high, solar storms and CMEs with strong magnetic field tend to sweep aside cosmic rays, making it more difficult for cosmic rays to reach Earth. Low solar activity with weaker magnetic field, on the other hand, allows an extra dose of cosmic rays to reach our planet.

In recent years, the cosmic ray environment around Earth has worsened. During the Solar Minimum of 2008-2009, the deepest in a century, the Sun and its solar wind exhibited extremely low densities and magnetic field strengths, representing states never before seen during the Space Age (Mewaldt et al., 2010; Schwadron et al., 2014b). As a result, cosmic ray levels jumped to their highest levels of the modern era. The anemic Solar Maximum of 2010-2014 provided little relief. Cosmic ray levels barely dropped as the solar cycle produced its weakest peak since the early 1900s.

The recent intensity of cosmic rays prompted Dr. Nathan Schwadron of University of New Hampshire and colleagues to publish a paper in 2014 entitled "Does the worsening galactic cosmic radiation environment observed by CRaTER preclude future manned deep space exploration?" (Schwadron et al., 2014b). Using data from a cosmic ray telescope onboard NASA's Lunar Reconnaissance Orbiter, they concluded that while increasing fluxes of cosmic rays "are not a show stopper for long duration missions (e.g., to the Moon, an asteroid, or Mars), galactic cosmic radiation remains a significant and worsening factor that limits mission durations."

Perhaps it is time that we begin to apply this kind of thinking to air travelers as well as astronauts. Many forecasters expect solar activity to drop sharply in the years ahead as we approach a new Solar Minimum. Cosmic rays are poised to increase accordingly. How many hours should pilots and flight attendants spend in the radiation zone? What are the possible health effects of intensifying radiation? How many people experience "everyday space weather" in this way?

At the moment, the last question is the only one we can answer: $\sim 3$ billion passengers per year.

\section{You have experienced "everyday space weather" if ... you have paid your electricity bill.}

Worldwide power grids are sensitive to solar storms. Since the beginning of the Space Age the total length of high-voltage power lines crisscrossing North America has increased nearly 10 fold. This has turned power grids into giant antennas for geomagnetically induced currents. With demand for power growing even faster than the grids themselves, modern networks are sprawling, interconnected, and stressed to the limit - a recipe for trouble, according to the National Academy of Sciences: "The scale and speed of problems that could occur on these modern grids have the potential to impact the power system in ways not previously experienced," they said in a 2008 report entitled "Severe Space Weather Events - Understanding Societal and Economic Impacts." A 2009 report by the North American Electric Reliability Corporation (NERC) and the US Department of Energy agreed that modern power systems have a "significantly enhanced vulnerability and exposure to effects of a severe geomagnetic storm."

The National Academy of Sciences report went even further, warning that a geomagnetic storm akin to the Carrington Events of 1859 could trigger widespread and long lasting power outages, with significant economic impacts. Multi-ton transformers damaged by such a storm might take years to repair.

Such a scenario hardly classifies as "everyday" space weather. Nevertheless the possibility of a superstorm has a ripple effect that we do feel everyday. We feel it in our wallets.

Prompted in part by the alarm-bells of the 2008 report, power companies have taken steps to "harden" their transformers against geomagnetically induced currents; they routinely train operators to respond to storm-time conditions and in the 
US this is now required through FERC Phase I GMD regulations; and they have "smartened the grid" to react to the shifting stresses of geomagnetic activity. All of these steps make the power grid safer and more reliable. They also add to the cost of electrical power.

Meanwhile, there is a dawning suggestion that big storms are not required to affect power grids. Relatively minor, "everyday" geomagnetic activity may be important, too. Geomagnetically induced currents far too small to cause a full-scale blackout may cause voltage sags, transients, and unwanted harmonics. This affects the quality of power and any equipment that happens to use such as computer servers, telecommunications equipment, generators, and motors. A joint Zurich Insurance - Lockheed Martin study correlated electrical insurance claims with geomagnetic activity and concluded that the economic impact of "everyday" space weather on the US and European economies amounted to more than 10 billion dollars per year (Schrijver et al., 2014). This one of a kind study makes it clear that many more studies of this nature need to be carried out in every sector of space weather impact. Other examples of such studies in Greece and South Africa indicate the global nature of every day space weather (Zois, 2013; Gaunt, 2014). For example the Zois (2013) study looked at the short term and long term effects of solar activity on the large transformers $(150 \mathrm{kV}$ and $400 \mathrm{kV})$ of the Greek national electric grid concluding that both short term (immediate) and long term impacts of solar activity onto large transformers in a mid-latitude country like Greece.

You have experienced "everyday space weather" if ... you have used a Global Navigation Satellite System (GNSS) tracker.

Extreme ultraviolet radiation from the Sun ionizes the top of Earth's atmosphere, creating a deep layer of ionized gas - the "ionosphere" - that bends and distorts GNSS radio signals. If the ionization were uniform and well-behaved, it would be easy to compensate for its distorting effects. However, the ionosphere is anything but well-behaved. Everyday terrestrial weather can affect the ionosphere too. Lightning is responsible for producing disturbances such as sprites, elves, and gigantic jets; heats the lower ionosphere. Gravity waves and acoustic waves generated deposit large amounts of energy and momentum in the upper atmosphere and additional gradients in temperature and density contribute to the dynamics of the situation (Lay et al., 2015). These irregularities interact with GNSS transmissions in several ways and gives the GNSS device "blurred vision."

In the United States alone, more than 200 million people carry GNSS-capable smart phones. Runners, hikers, drivers i.e., people from all walks of life - use GNSS navigation on a daily basis to measure their own speed, to find out where they are going, or to simply find themselves.

Typical commercial GNSS units have an uncertainty of approximately $5 \mathrm{~m}$ or $16 \mathrm{ft}$. GNSS uncertainty is regional with near equatorial and polar hot spots and also platform dependent. But it would not be a stretch to say that about a third of this error is caused by everyday space weather.

The ionosphere expands during the day, and shrinks at night, breathing in and out. Layers of the ionosphere are only partially ionized and that too not-uniformly, creating nonuniform patches that are carried around by high altitude winds.
Gradients in temperature and density contribute to the dynamics of the situation.

These irregularities interact with GNSS transmissions in several ways. Large-scale irregularities refract GNSS signals, causing unexpected phase shifts that can challenge the tracking loops in GNSS receivers. Smaller irregularities, especially on scales near $400 \mathrm{~m}$, scatter GNSS signals, causing radio waves to reach the receiver through multiple paths. This, in a sense, gives the GNSS device "blurred vision." The same process occurs with light and can be seen in the fuzzy image passing through jet exhaust from a commercial airliner. Collectively, refractive and scattering effects are called "scintillation."

While flares may have a much stronger association with Sudden Increase in Total Electron Content (SITEC), signal scintillation of GNSS transmissions occurs every hour of every day. The problem worsens during Solar Max when frequent solar flares hit the ionosphere with sudden and unpredictable pulses of ionizing radiation. But it never goes away. Data from the CINDI (Coupled Ion-Neutral Dynamics Investigations) satellite, which probed the ionosphere from Earth orbit from 2008 until 2015, found richly textured irregularities during Solar Minimum as well as Solar Max.

During the deep solar minimum of 2008, the ionosphere surprised researchers by collapsing to record-low altitudes, some $200 \mathrm{~km}$ lower than predicted by the best physical models. They wouldn't have known except that the CINDI satellite was launched, luckily, into the deepest part of the extreme solar minimum. The satellite also recorded an unexpected drop in the temperature of the ionosphere. These changes alter the stage upon which scintillation occurs - it happens at a lower altitude, with colder plasma, with a different mix of ions and whole host of other conditions every day (Knipp, 2017).

Very High Frequency (VHF)/High Frequency (HF) radio propagation is another element of everyday space weather. HF radio propagation is a key technology for long distance communications, actively used by aircraft, by ships at sea, in military operations, for disaster relief efforts, and by amateur radio operators. There are about 3 million HAM (amateur radio operators) radio operators worldwide.

You have experienced the everyday effects of space weather if ... you have ever taken an aurora vacation.

"Space weather tourism" is an industry which operates through 6 months of the year and all phases of the solar cycle. During northern winter, people travel to Alaska, Iceland, and Scandinavia to take "Northern Lights tours." Industry estimates suggest that of order 10,000 people per year travel to the Arctic for aurora tours. With each tourist typically spending more than $\$ 5,000$ apiece in combined air fare, lodging, and tour fees, that makes it a 50+ million dollar a year industry.

Aurora tour guides do not rely on big solar storms. The auroras their customers come to see are often substorms or quiet-time auroras that occur when the planetary $K$-index is only 2 or 3. Even a dull night in Troms $\varnothing$, Norway, can be a wonderful thing to behold. In this way, tourism is a good example of everyday space weather (The New Your Times).

But not just space weather tourism, new space weather science is also a big beneficiary to this every day collection of citizen science data on auroral phenomenon. Citizen 
scientists have led to discovery of new phenomenon like STEVE (Strong Thermal Emission Velocity Enhancement) and "The Dunes" (Gallardo-Lacourt et al., 2018; Palmroth et al., 2020).

In summary, space weather is something ordinary people grapple with, enjoy, and pay for on a daily basis - no "super storms" required (Guhathakurta \& Phillips, 2013). We need to start discussing space weather as if it is an everyday occurrence. Because it is.

Acknowledgements. The author thanks Delores Knipp and Irina Kitashvili for providing valuable comments on the manuscript. The views expressed in this article are those of the author. The editor thanks two anonymous reviewers for their assistance in evaluating this paper.

\section{References}

Bolduc L. 2002. GIC observations and studies in the Hydro-Quebec power system. J Atmos Sol Terr Phys 64: 1793-1802.

Connick DE, Smith CW, Schwadron NA. 2011. Interplanetary magnetic flux depeletion during protracted solar minima. Astrophys J 727: 8-14. https://doi.org/10.1088/0004637X/727/1/8.

Gallardo-Lacourt B, Liang J, Nishimura Y, Donovan E. 2018. On the origin of STEVE: Particle precipitation or ionospheric Skyglow? Geophys Res Lett 45(16): 7968-7973. https://doi.org/10.1029/ 2018 GL078509.

Gaunt CT, Coetzee G. 2007. Transformer failures in regions incorrectly considered to have low GIC-risk. In: Power Tech, 2007 IEEE Lausanne, pp. 807-812.

Gaunt CT. 2014. Reducing uncertainty - responses for electricity utilities to severe solar storms. J Space Weather Space Clim 4: A01. https://doi.org/10.1051/swsc/2013058.

Gopalswamy N, Akiyama S, Yashiro S, Xie H, Mäkelä P, Michalek G. 2014. Anomalous expansion of coronal mass ejections during solar cycle 24 and its space weather implications. Geophys Res Lett 41: 2673. https://doi.org/10.1002/2014GL059858.

Gopalswamy N, Akiyama S, Yashiro S, Xie H, Mäkelä P, Michalek G. 2015. The mild space weather in solar cycle 24 . In: $14^{\text {th }}$ International Ionospheric Effects Symposium on "Bridging the gap between applications and research involving ionospheric and space weather disciplines" May 12-14, 2015, Alexandria, VA. ArXiv e-prints, arXiv:1508.01603.

Guhathakurta M, Phillips T. 2013. The solar cycle turned sideways. Space Weather 11: 212-213. https://doi.org/10.1002/swe.20039.

Knipp DJ. 2017. Essential science for understanding risks from radiation for airline passengers and crews. Space Weather 15: 549-552. https://doi.org/10.1002/2017SW001639.

Lay EH, Shao XM, Kendrick AK, Carrano CS. 2015. Ionospheric acoustic and gravity waves associated with midlatitude thunderstorms. JGR Space Phys 120: 6010-6020. https://doi.org/10.1002/ 2015JA021334.

McComas DJ, Ebert RW, Elliott HA, Goldstein BE, Gosling JT, Schwadron NA, Skoug RM. 2008. Weaker solar wind from the polar coronal holes and the whole Sun. Geophys Res Lett 35: L18103. https://doi.org/10.1029/2008GL034896.
McComas DJ, Angold N, Elliott HA, Livadiotis G, Schwadron NA, Skoug RM, Smith CW. 2013. Weakest solar wind of the space age and the current "Mini" solar maximum. Astrophys $J$ 779: 2. https://doi.org/10.1088/0004637X/779/1/2.

Mertens CJ, Gronoff GP, Norman RB, Hayes BM, Lusby TC, et al. 2016. Cosmic radiation dose measurements from the RaD-X flight campaign. Space Weather 14: 874-898. https://doi.org/10.1002/ 2016 SW001407.

Mewaldt RA, Davis AJ, Lave KA, Leske RA, Stone EC, et al. 2010. Record-setting cosmic-ray intensities in 2009 and 2010. Astrophys J Lett 723: L1-L6. https://doi.org/10.1088/2041-8205/723/1/L1. https://www.nytimes.com/2019/02/11/travel/northern-lights-tourismin-sweden.html.

Palmroth M, Grandin M, Helin M, Koski P, Oksanen A, et al. 2020. Citizen scientists discover a new auroral form: Dunes provide insight into the upper atmosphere. $A g u A d v$ 1: 1. https://doi.org/ 10.1029/2019AV000133.

Pulkkinen A, Lindahl S, Viljanen A, Pirjola R. 2005. Geomagnetic storm of 29-31 October 2003: Geomagnetically induced currents and their relation to problems in the Swedish high-voltage power transmission system. Space Weather 3: S08C03. https://doi.org/ 10.1029/2004SW000123.

Schrijver CJ. 2015. Socio-economic hazards and impacts of Space Weather: The important range between mild and extreme. Space Weather 13: 524-528. https://doi.org/10.1002/2015SW001252.

Schrijver CJ, Dobbins R, Murtagh W, Petrinec SM. 2014. Assessing the impact of space weather on the electric power grid based on insurance claims for industrial electrical equipment. Space Weather 12: 487-498. https://doi.org/10.1002/2014SW001066.

Schwadron NA, McComas DJ. 2008. The solar wind power from magnetic flux. Astrophys J 686: L33-L36. https://doi.org/10.1086/ 592877.

Schwadron NA, Goelzer ML, Smith CW, Kasper JC, Korreck K, Leamon RJ, Lepri ST, Maruca BA, McComas D, Steven ML. 2014a. Coronal electron temperature in the protracted solar minimum, the cycle 24 mini maximum, and over centuries. J Geophys Res (Space Phys) 119: 1486-1492. https://doi.org/10.1002/2013JA019397.

Schwadron NA, Blake JB, Case AW, Joyce CJ, Kasper J, et al. 2014b. Does the worsening galactic cosmic radiation environment observed by CRaTER preclude future manned deep space exploration Space Weather 11: 622-632. https://doi.org/10.1002/ 2014SW001084.

Schwadron NA, Rahmanifard F, Wilson J, Jordan AP, Spence HE, Joyce CJ, Blake JB, Case AW, de Wet W, Farrell WM, Kasper JC, Looper MD, Lugaz N, Mays L, Mazur JE, Niehof J, Petro N, Smith CW, Townsend LW, Winslow R, Zeitlin C. 2018. Update on the worsening particle radiation environment observed by CRaTER and implications for future human deep-space exploration. Space Weather 16: 289-303. https://doi.org/10.1002/2017SW001803.

Tobiska WK, Bouwer D, Smart D, Shea M, Bailey J, et al. 2016. Global real-time dose measurements using the Automated Radiation Measurements for Aerospace Safety (ARMAS) system. Space Weather 14: 1053-1080. https://doi.org/10.1002/2016SW001419.

Zois IA. 2013. Solar activity and transformer failures in the Greek national electric grid. J Space Weather Space Clim 3: A32. https://doi.org/10.1051/swsc/2013055. 